\title{
THE NOVEMBER MEETING IN EVANSTON
}

The four hundred ninety-seventh meeting of the American Mathematical Society included a Symposium on Special Topics in Applied Mathematics and was held on Friday and Saturday, November 2728,1953 . The Symposium was sponsored jointly by the Society and the National Research Council, under a contract with the National Science Foundation. There was a total registration of 256 , including the following 206 members of the Society:

E. S. Allen, W. R. Allen, J. M. Anderson, D. L. Arenson, E. L. Arnoff, Nachman Aronszajn, Robert Baer, R. H. Bardell, W. E. Barnes, R. B. Barrar, A. F. Bausch, G. E. Baxter, J. W. Beach, A. A. Blank, H. D. Block, G. M. Bloom, L. M. Blumenthal, R. P. Boas, W. M. Boothby, G. U. Brauer, Leonard Bristow, F. E. Browder, R. H. Bruck, H. D. Brunk, R. C. Buck, E. L. Buell, R. S. Burington, James William Butler, S. S. Cairns, K. H. Carlson, J. W. Carr, W. B. Caton, Lamberto Cesari, Subrahmanyan Chandrasekhar, Y. W. Chen, S. S. Chern, Gustave Choquet, H. M. Clark, M. D. Clement, L. W. Cohen, Harvey Cohn, S. H. Cohn, Richard Courant, V. F. Cowling, C. W. Curtis, H. J. Curtis, M. L. Curtis, W. F. Darsow, R. B. Deal, Jr., John DeCicco, J. C. E. Dekker, R. J. DeVogelaere, J. A. Dieudonné, N. J. Divinsky, C. L. Dolph, M. D. Donsker, R. J. Driscoll, W. L. Duren, Jr., Meyer Dwass, W. F. Eberlein, J. D. Elder, Robert Ellis, Paul Erdös, H. P. Evans, R. L. Evans, Trevor Evans, G. M. Ewing, Chester Feldman, D. A. Flanders, H. H. Fox, W. C. Fox, J. S. Frame, Evelyn Frank, H. D. Friedman, C. G. Fry, R. E. Fullerton, R. A. Gambill, H. L. Garabedian, P. R. Garabedian, J. J. Gergen, J. H. Giese, David Gilbarg, Leonard Gillman, Casper Goffman, H. E. Goheen, Bernice Goldberg, Malcolm Goldman, Michael Golomb, A. W. Goodman, L. M. Graves, R. L. Graves, L. W. Green, Harold Greenspan, L. W. Griffiths, P. E. Guenther, M. M. Gutterman, T. E. Hagensee, J. K. Hale, P. R. Halmos, P. C. Hammer, L. J. Heider, Melvin Henriksen, E. H. C. Hildebrandt, D. L. Holl, T. C. Holyoke, Eberhard Hopf, B. E. Howard, W. A. Howard, K. C. Hsu, H. K. Hughes, Ralph Hull, A. W. Jacobson, Arno Jaeger, T. J. Jaramillo, W. E. Jenner, E. R. Johnston, Mark Kac, Samuel Kaplan, Leo Katz, N. D. Kazarinoff, D. E. Kearney, R. B. Kellogg, L. M. Kelly, D. E. Kibbey, S. C. Kleene, Jacob Korevaar, P. H. Kratz, A. H. Kruse, M. Z. Krzywoblocki, C. D. LaBudde, H. G. Landau, R. E. Lane, Serge Lang, R. E. Langer, E. H. Lee, G. R. Lehner, Joseph Lehner, Jean Leray, Frank Levin, J. J. Levin, G. G. Lorentz, Saunders MacLane, H. M. MacNeille, Morris Marden, E. P. Merkes, B. E. Meserve, A. N. Milgram, M. D. Morley, W. L. Murdock, Edward Nelson, R. J. Nunke, R. H. Oehmke, H. A. Osborn, L. G. Peck, Pasquale Porcelli, G. B. Price, A. L. Putnam, W. T. Reid, Haim Reingold, R. B. Reisel, P. C. Rosenbloom, M. A. Rosenlicht, Arthur Rosenthal, E. H. Rothe, L. A. Rubel, Hans Samelson, O. F. G. Schilling, Morris Schreiber, W. T. Scott, D. H. Shaftman, M. E. Shanks, J. M. Shapiro, Edward Silverman, R. J. Silverman, H. T. Slaby, F. B. Sloss, J. M. Slye, D. M. Smiley, M. F. Smiley, Jerome Spanier, C. E. Springer, George Springer, W. F. Stinespring, A. H. Taub, J. S. Thale, E. A. Trabant, J. W. Tukey, H. L. Turrittin, C. J. Vanderlin, Jr., Bernard Vinograde, R. D. Wagner, G. L. Walker, Daniel Waterman, L. M. Weiner, C. P. Wells, F. J. Weyl, George Whaples, L. R. Wilcox, G. N. Wollan, F. B. Wright, Jr., F. M. Wright, A. W. Wymore, L. C. Young, J. W. T. Youngs, Daniel Zelinsky, J. L. Zemmer, Jr. 
The Committee to Select Hour Speakers for Western Sectional Meetings had invited Professor P. C. Rosenbloom of the University of Minnesota to address the Society. Professor Rosenbloom spoke on The heat equation on Saturday, November 28, at 10:30 A.M. The presiding officer was Professor R. P. Boas.

The Symposium was divided into three sessions which met respectively at 10:15 A.M. and 1:30 P.M. on Friday and at 1:30 P.M. on Saturday. The first one was presided over by Professor Richard Courant of New York University, and papers by Professor Jean Leray of the Institute for Advanced Study and the College de France on The physical fact and the differential equations, by Dr. L. G. Peck of New York University on One dimensional shock-wave flows and by Professor P. R. Garabedian of Stanford University on Recent advances in the application of conformal mapping to hydro-dynamics were read.

The second session with Professor J. W. Tukey of Princeton University in the chair introduced Professor D. E. Muller of the University of Illinois, Professor Mark Kac of Cornell University, and Professor S. S. Cairns of the University of Illinois, who spoke respectively on Boolean algebras in electric circuit design, Signal and noise problems and Discrete structures and large-scale computers.

The topics of the final session were Problems of astro-physical fluid dynamics, The theory of lattice vibrations, and The geometric structure of shock-wave configurations, presented respectively by Professor Subrahmanyan Chandrasekhar of the University of Chicago, Professor E. W. Montroll of the University of Maryland and the Office of Naval Research, and Professor A. H. Taub of the University of Illinois, Dr. F. J. Weyl, Office of Naval Research and the National Research Council, presiding.

Sessions for the presentation of contributed papers were held on both Friday and Saturday at 9:00 A.M. and 3:30 P.M. Presiding officers at these sessions were Dr. W. E. Jenner and Professors G. M. Ewing, L. M. Blumenthal and W. T. Reid, respectively.

There was a dinner for members of the Society and their guests in Sargent Hall on Friday evening, at which Professor H. T. Davis acted as toastmaster.

Abstracts of papers presented at the meeting are recorded below. Abstracts whose numbers are followed by " $t$ " were read by title. In the case of joint papers presented in person, (p) after a name indicates the author who read the paper. Mr. Gordon was introduced by Professor Antoni Zygmund, Professor Halperin by Professor Norman Miller, Dr. Junkat and Professor Knopp by Professor C. N. Moore. 


\section{Algebra AND Theory of Numbers}

102. R. H. Bruck and H. T. Slaby (p): Central nilpotency of commutative Moufang loops.

Let $G$ be a commutative Moufang loop. The associator $(x, y, z)$ of elements $x, y, z$ $\in G$ is defined by $x y \cdot z=(x \cdot y z)(x, y, z)$. If $H, K, N$ are normal subloops of $G$, let $(H, K, N)$ denote the (normal) subloop of $G$ generated by the set of associators $(h, k, n), h \in H, k \in K, n \in N$. The lower central series of $G$ is the sequence $\left\{G_{i}\right.$; $i=0,1,2, \cdots\}$ defined by $G_{0}=G, G_{i+1}=\left(G_{i}, G, G\right)$. For all non-negative integers $i, j, k,\left(G_{i}, G_{i}, G_{k}\right) \subseteq G_{i+j+k+1}$. If $G_{k}=1$ and $k$ is the least integer for which this is true, then $G$ is said to be centrally nilpotent of class $k$. For $n=3,4,5$, if $G$ is generated by $n$ elements, then $G$ is centrally nilpotent of class at most $n-1$. (Received October 13, 1953.)

\section{3t. K. T. Chen: On a Cartan's theorem and its converse.}

First a local Lie group is constructed from a given Lie algebra by using Hausdorff's formula $(\exp x)(\exp y)=\exp z$, where $x$ and $y$ are noncommutative indeterminates and $z$ is an infinite sum of bracket products of $x$ and $y$. This leads immediately to the Cartan's theorem that, in a canonical coordinate system of the first kind in a nilpotent local Lie group $G$, the multiplication is defined by polynomials, i.e., if $(\alpha)$ $=\left(\alpha^{1}, \cdots, \alpha^{n}\right)$ and $(\beta)=\left(\beta^{1}, \cdots, \beta^{n}\right)$ are two points of $G$, then each coordinate of their product $(\alpha) \cdot(\beta)$ is a polynomial in the $\alpha$ 's and $\beta$ 's. The converse of this theorem is proved by using a theorem due to Engel and the formula $\log \{(\exp -x)(\exp y)(\exp x)\}=y+[y, x]+[[y, x], x] / 2 !+[[[y, x], x], x] / 3 !+\cdots$. (Received September 23, 1953.)

\section{4t. Harvey Cohn: Stability properties of cyclotomic units.}

Completing results indicated in an earlier abstract (Bull. Amer. Math. Soc. Abstract 58-6-571) the author considers fields for which infinitesimal variations of the basis preserving the discriminant (determinant) will decrease the norms of the units. These so-called stable fields include all nontrivial $R(\cos 2 \pi / N)$ (except $N=12$ ), as well as just those $R(\exp 2 \pi i / N)$ for which $N$ is square-free. The first result depends essentially on the fact that the smaller half of the absolute residues $(\bmod N)$ are not closed under multiplication. The second result depends on the existence of a normal basis for the field. (Research sponsored by the U.S. Army Office of Ordnance Research under contract DA-20-018-ORD-12332.) (Received October 9, 1953.)

\section{C. W. Curtis: $A$ note on the representations of nilpotent Lie} algebras.

The following theorems are proved. (1) Let $x \rightarrow U_{x}$ be an indecomposable representation of a nilpotent Lie algebra $\mathfrak{R}$ over a field $K$. Then for all $x \in \mathfrak{R}$, the minimum polynomial of $U_{x}$ is a prime power. Moreover, any two irreducible constituents of $U$ are equivalent. (2) Let $x \rightarrow U_{x}$ be an irreducible representation of a Lie algebra $\&$ over a field $K$ of characteristic $p>0$. If $\mathfrak{D}$ is a Lie algebra of derivations acting in $\mathbb{R}$, then there exists an irreducible representation $x \rightarrow T_{x}$ of the semi-direct sum $\mathbb{R}+\mathfrak{D}$, and a 1-1 linear transformation $S$ of the representation space of $U$ into the representation space of $T$ such that $S T_{x}=U_{x} S$ for all $x \in \mathbb{R}$. (3) If $\&$ is a nilpotent Lie algebra over a 
field $K$ of characteristic $p>0$, and if $\left(x_{1}, \cdots, x_{n}\right)$ is a basis of $\mathbb{R}$ such that $\left[x_{i} x_{i}\right]$ $\in\left(x_{i+1}, \cdots, x_{n}\right)$, then for each set $\left(f_{1}, \cdots, f_{n}\right)$ of irreducible polynomials in $K[X]$, there exists one and only one irreducible representation $U$ of $\mathbb{R}$ such that the minimum polynomial of $U_{x_{i}}$ is a power of $f_{i}, i=1, \cdots, n$. Theorem (3) is an extension to arbitrary fields of prime characteristic of a theorem of Zassenhaus (Abh. Math. Sem. Hamburgischen Univ. vol. 13 (1939)). The ideal-theoretic properties of the universal associative algebra are exploited in the proofs of (2) and (3). (Received October 13, 1953.)

\section{N. J. Divinsky: Pseudo-regularity.}

An element $x$ of a ring $A$ is called right-pseudo-regular (r.p.r.) of degree $n$ if there exists an element $y$ such that $x+x^{n} y+x^{n+1} y=0$. A study is made of the case $n=1$, and the existence of a maximal r.p.r. ideal $P$ and the existence of a maximal left p.r. ideal $P_{L}$ are established. It is shown that in general they are different from each other and smaller than the Perlis-Jacobson radical $R$. Several radical-like properties are established and in the commutative case with descending chain conditions a definite relationship is obtained between $P, P_{L}$, and $R$. The techniques of Brown and $\mathrm{McCoy}$ are used to discover the structure of commutative rings modulo their subradicals $(P)$. Finally the cases $n>1$ are studied. Use is made of an extension of McCoy's result on commutative subdirectly irreducible rings, namely, that every commutative subdirectly irreducible ring, all of whose elements are divisors of zero, is bound to its maximal nil ideal as well as to its Perlis-Jacobson radical (in the sense of Hall). (Received September 24, 1953.)

\section{7t. S. I. Goldberg: Lie algebra extensions and enlargements of} modules. II.

Let $(E, K, \phi, u$ ) be a $K$-extension of the Lie algebra $G$ by the $G$-module $M$ with factor set $g$ and $(\epsilon, K, \psi, \mu)$ a $K$-enlargement of the $G$-module $P$ by $M$. Then $\gamma \cdot \mu_{m}$ $=\mu_{\gamma . m}+\beta_{\gamma}(m), \gamma \in G, m \in M, \mu_{m} \in \epsilon, \beta \in C^{1}(G, K, \operatorname{Hom}(M, P)), \delta \beta=0$ where the elements $\mu_{m}$ are the representatives corresponding to the isomorphism $\epsilon / P \approx M$. It is well known that the sequence $\cdots \rightarrow H^{i-1}(G, M) \rightarrow{ }^{\Delta} H^{i}(G, P) \rightarrow H^{i}(G, \epsilon) \rightarrow H^{i}(G, M)$ $\rightarrow \cdots$ is exact. The invariant coboundary $\Delta$ is described as follows: Let $\psi: \epsilon \rightarrow M$ be the given homomorphism of $\epsilon$ upon its quotient $\epsilon / P$, and $g$ be any cocycle in $C^{i-1}(G, M)$. Select representatives $\bar{g}\left(\gamma_{1}, \cdots, \gamma_{i-1}\right)$ at random so that $\bar{g}$ is multilinear and $\psi \bar{g}\left(\gamma_{1}, \cdots, \gamma_{i-1}\right)=g\left(\gamma_{1}, \cdots, \gamma_{i-1}\right)$. Then $f=\delta \tilde{g}$ has values in $P$ and the mapping $\Delta$ is the one obtained by sending the cohomology class of $g$ in $H^{i-1}(G, M)$ into that of $f$ in $H^{i}(G, P)$. It is shown that a necessary and sufficient condition for the existence of a $K$-extension $\epsilon^{*}$ of $G$ by $\epsilon$ with $\epsilon^{*} / P \approx E$ is $\Delta(g)=\delta \bar{g}, \bar{g} \in C^{2}(G, K, \epsilon)$, that is, $\Delta(g)$ is a relative coboundary modulo $K$. (Received October 13, 1953.)

\section{Arno Jaeger: Riccati's differential equation in fields of char-} acteristic 2.

While Riccati's differential equation in a field $F$ of characteristic $p>2$ can always be solved in a quadratic extension of $F$ (cf. F. Kasch, Arch. Math. vol. 4 (1953) pp. $17-22)$ the case $p=2$ presents a different situation: If $D$ is a regular differentiation in the sense of A. Jaeger (Monatshefte fur Mathematik vol. 56 (1952) p. 189) the differential equation (1) $R(y) \equiv D y+a y^{2}+b y+c=0(a, b, c \in F ; a \neq 0)$ implies (2) $D(R(y)) \equiv b D y+(D a) y^{2}+(D b) y+D c=0$. By elimination of $D y$ resp. $y^{2}$ from (1) and (2) one obtains an at most quadratic equation in $y$ resp. a linear differential equation 
in $y$, and it has to be checked how far their common solutions solve (1), too. With the abbreviation $P(y)=b y+D y$ one obtains: (a) If $P(b) \neq 0$ or if $P(b)=P(a)=P(c)=0$ there exist two distinct solutions in a separable extension of $F$ or in $F$. (b) If $P(b)=0$ and $P(a) \neq 0$ there is at most one solution, possibly in an inseparable, but $D$-admissible, extension of $F$, and the vanishing of a certain differential polynomial of order 3 in $a, b, c$ is necessary and sufficient for solvability. (c) If $P(b)=P(a)=0$, but $P(c) \neq 0$, there is no solution. (Received November 28, 1953.)

\section{9t. A. R. Schweitzer: On the transition from Grassmann's exten- sive algebra to modern number theory.}

In his article, Applications of Grassmann's extensive algebra (Amer. J. Math. vol. 1, pp. 350-358) W. K. Clifford has developed a system of complex units. In his treatise, Untersuchungen ueber die Summen von Quadraten (Bonn, 1886) R. Lipschitz asserts (p. 2) that a system of units due to himand reported in C. R.Acad.Sci. Paris (October, 1880) agrees with Clifford's system. In this paper the author interprets Lipschitz's article in the Comptes Rendus as an introduction to the treatise mentioned above. In the latter treatise Lipschitz successively aims to determine all linear transformations with rational coefficients which leave invariant (1) the sum of two squares, (2) the sum of three squares, (3) the sum of $n$ squares $(n>3)$; see pp. 7, 54, 79. To attain his object Lipschitz uses respectively as a tool (1) Gauss's theory of integral complex numbers, (2) a theory of integral quaternions, (3) a theory of integral complex expressions of the $n$th order $(n>3)$. In his treatise Lipschitz discusses (p. 26) a genesis of quaternions to which the author makes transition from his determination of the quaternion in Grassmann's extensive algebra (Math. Ann. vol. 69 (1910)). An interesting commentary on Lipschitz's theory of integral quaternions is due to L. E. Dickson, Algebras and their arithmetics (Chicago, 1923). (Received October 14, 1953.)

110. L. M. Weiner (p) and Pasquale Porcelli: $A$ derivation of Cauchy's inequality for polynomials.

The following lemmas and theorem are established: Lemma 1: If $m-1$ is a positive integer, $r_{1}=1, r_{2}, \cdots, r_{m}$ the $m m$ th roots of unity, $p$ a positive integer less than $m+1, s$ a positive integer less than $m$ such that $T_{s}$ denotes the sum of the products of the $r_{i}$, excluding $r_{p}$, taken $s$ at a time, then $T_{s}$ has absolute value 1. Lemma 2: Under the hypotheses of Lemma $1, \prod_{i=1, i \neq p}^{i=m}\left|r_{p}-r_{i}\right|=m$. Theorem: If $n$ is a positive integer, $\left\{a_{p}\right\}_{p=0}^{n}$ a sequence of numbers, and each of $M$ and $R$ a positive number such that $\left|\sum_{p-0}^{n} a_{p} z^{p}\right|<M$ for $z=R e^{2 \pi i t / n+1}$ and $t=1,2, \cdots, n+1$, then $\left|a_{p}\right|$ $<M / R^{p}$ for $p=0,1, \cdots, n$. (Received October 9,1953 .)

\section{ANALysis}

111. Nachman Aronszajn: Linear functionals on proper functional spaces and reproducing kernels. Applications to Green's functions.

Linear functionals $L(f)$ on a proper functional space $\mathcal{F}$ on a basic set $\mathcal{E}$ are always limits of finite linear combinations $L_{S}(f)=\sum \alpha_{k} f\left(x_{k}\right)$ for systems $S=\left\{x_{k} ; \alpha_{k}\right\}, x_{k} \in \mathcal{E}$, $\alpha_{k}$ real or complex. If $\mathcal{F}$ is a Hilbert space, $K(x, y)$ its reproducing kernel, and $L$ a linear functional given as limit of $L_{S}$, then a necessary and sufficient condition in order that $L(f)$ exists and be continuous on $\mathcal{F}$ is that $L_{s}^{(x)} L_{s}^{(y)} K(x, y)$ converge to a finite limit $L^{(x)} L^{(y)} K(x, y)$. If we have in $\mathcal{F}$ two equivalent quadratic norms, $\|f\|$ and $\|f\|_{1}$, the above property for the corresponding kernels $K$ and $K_{1}$ will be simultaneously 
true or false. This allows us to prove regularity properties of Green's functions and their derivatives for a large class of differential problems. (Received October 15,1953.)

112. F. E. Browder: The eigenfunction expansion theorem for the general singular self-adjoint elliptic system of differential operators.

Let $D$ be a domain in Euclidean $n$-space (or in a sufficiently differentiable manifold of dimension $n$ ). Suppose that $K$ is a formally self-adjoint system of $r$ differential operators of order $t$ acting on $r$-vectors and with coefficients defined on $D$. Suppose that on every compact subdomain of $D, K$ is elliptic and suitably differentiable. Let $K_{0}$ be the symmetric operator in the $r$-vector $L^{2}(D)$ obtained by restricting $K$ to the vector functions whose components are infinitely differentiable and vanish outside compact subsets of $D$. Suppose that $K_{1}$ is a self-adjoint extension of $K_{0}$. Then, it is shown that $K_{1}$ has an eigenfunction expansion in the sense defined by $\mathrm{F}$. Mautner (Proc. Nat. Acad. Sci. U.S.A. (1953) pp. 49-53) and the properties of this expansion are discussed. The basic part of the proof rests in showing for $\operatorname{Im}(c) \neq 0, k>n / 2 t$, that $\left(K_{1}-c I\right)^{-k}$ is a bounded system of integral operators of Carleman type. The theorem contains as a special case the results of Weyl, Kodaira, and others on eigenfunction expansions for systems of ordinary differential equations. (Received October 9, 1953.)

\section{Gustave Choquet: On a class of set-functions.}

The author studies the properties of positive and subadditive capacities defined on an additive and hereditary set of compact subsets of a space $E$. (1) Several conditions equivalent to the fact that the intersection of two capacitable sets is always capacitable are given: strict increase of $f$ is, roughly speaking, the condition in order that this be verified. (2) Comparison of several classes of subsets of $E$, the approximable, perfectly capacitable and accessible subsets whose interest is their invariance under most of the ordinary operations: difference, union, and intersection (finite or denumerable). (3) Detailed study of the simple capacities $f$ defined as follows: If $\phi$ is a continuous application of $E$ into $F$ measured by $\mu \geqq 0$, for every compact $K \subset E$, let $f(K)=\mu(\phi(K))$. When $E$ and $F$ are compact and metric, and $f$ is of finite total variation, $f$ can be considered as a superposition of a sequence of Radon measures, and there is identity between accessible, approximable, and perfectly capacitable subsets. (Received October 15, 1953.)

\section{R. A. Gambill: Criteria of stability and instability for linear sys-} tems of differential equations with periodic coefficients.

Consider the differential system (A) $y_{i}^{\prime \prime}+\sigma_{i}^{2} y_{i}+\lambda \sum_{h=1}^{n} \psi_{i h}(x) y_{h}=0, i=1, \cdots, n$, where $\lambda$ is a small real parameter, $\sigma_{1}, \cdots, \sigma_{n}$ are distinct positive numbers, and (1) $\psi_{i h}(x)$ are real periodic functions of period $T=2 \pi / \omega$, of mean value zero; (2) $\psi_{i h}(x)$ have absolutely convergent Fourier series; (3) $m \omega \neq \sigma_{i} \pm \sigma_{j}, i, j=1, \cdots, n ; m$ $=0,1, \ldots$. The solution (B) $\left[y_{i} \equiv 0, i=1, \cdots, n\right]$ of $(\mathrm{A})$ is said to be parametrically stable (Liapounoff) if, given $\epsilon>0$, there exists a $\delta>0$ such that $|\lambda|<\delta, \mid y_{i}(0$, $\lambda) \mid<\delta$ implies $\left|y_{i}(t, \lambda)\right|<\epsilon$ for all $t \geqq 0$ [or $t \leqq 0$ ]. If (4) $\left\|\psi_{i h}\right\|=\left\|a_{i h}\right\|+\left\|b_{i h}\right\|$, where $\left\|a_{i n}\right\|=\operatorname{diag}\left(A_{1}, A_{2}, \cdots, A_{k}\right), b_{i h}$ may be $\neq 0$ only if they are all below [or above] the matrices $A_{i} ;(5)$ for each $A_{i}=\left\|\psi_{\mu \nu}\right\|$, either $A_{i}$ is symmetric or the functions $\psi_{\mu \nu}$ are even, then (B) is parametrically stable (in both directions). In particular, conditions (4), (5) are satisfied if $\psi_{i h} \equiv 0$ for all $i<h[i>h]$. This theorem is proved by means of a variant, studied by L. Cesari [Mem. Accad. Italia (4) vol. 11, pp. 634692], of Poincaré's method of casting out the secular terms in the solution of (A). 
For a given system (A), it may happen that the solution (B) is nonparametrically stable for every $\omega$. Criteria are given for this behavior. These criteria state that if any of certain expressions $P, Q, \cdots$, depending on $\psi_{i h}$ and the numbers $\sigma_{i}$, are $\not \equiv 0$, then necessarily this is the expected behavior. Thus these criteria show that this behavior is rather the rule. Condition (2) can be replaced by $L$-integrability in $(0, T)$ (J. K. Hale). (Received October 13, 1953.)

\section{5t. P. R. Garabedian and Max Shiffman: On the solution of partial} differential equations by the Hahn-Banach theorem.

The following construction of the Green's function and the Neumann's function for the elliptic partial differential equation $\Delta u-p u=0$ in a domain $D$ of $n$-dimensional space is developed, where $p$ is a positive, suitably regular function. Consider the class of functions $v$ in $D$ which vanish on the boundary of $D$ and have continuous second derivatives in $D$. For $z$ in $D$, let $L$ denote the linear functional defined by the formula $v(z)=L[\Delta v-p v]$ over the space of functions of the form $\Delta v-p v$. By the maximum principle, the functional $L$ is bounded, and it can therefore be extended to the whole space of continuous functions in $D$. Let $S(z, w)$ be a parametrix for $\Delta u-p u$ $=0$ which vanishes on the boundary of $D$. It is shown that $G=S-L[\Delta S-p S]$ is the desired Green's function. This existence proof has broader applications than the earlier one due to Lax (Proc. Amer. Math. Soc. vol. 3 (1952) pp. 526-531) because only a parametrix, and not a fundamental solution, is required in the construction. (Received October 5, 1953.)

\section{A. W. Goodman: On regions omitted by univalent functions. II.}

Let $S$ denote the family of functions $f(z)=z+a_{2} z^{2}+\cdots$ regular and univalent in $|z|<1$, let $A_{f}$ denote the area of the intersection of the circle $|w|<1$, and the image of $|z|<1$ under $f(z)$, and let $A=\inf _{f} \in s\left\{A_{f}\right\}$. Jenkins (Amer. J. Math. vol. 75 (1953) pp. 406-408) has proved that $A>.5387 \pi$. With a slight refinement of Jenkins' methods, it is possible to prove that $A>.6023 \pi$. It is known that $A<.7728 \pi$. (Received August 3, 1953.)

\section{7t. L. I. Gordon: Fractional anti Laplacians. Preliminary report.}

Consider functions $f(p)=f(x, y)$ of two real variables, periodic (say, of period 1) in $x$ and $y$. By $L^{r}$, denote the class of functions $f$ such that $|f| r$ is integrable over the unit square. Then for $\beta>0, \sum_{m, n}^{\prime}\left(m^{2}+n^{2}\right)^{-\beta / 2}(2 \pi i)^{-\beta} e^{2 \pi i(m x+n y)}$ is the Fourier series of a function $\psi_{\beta}(x, y) \in L\left(=L^{\prime}\right)$. Let $f \in L, f \sim \sum_{m, n} c_{m n} e^{2 \pi i(m x+n y)}$, and let $I_{\beta} f$ denote the convolution of $f$ with $\psi_{\beta}$. Then $I_{\beta} f \sim \sum_{m, n}\left(m^{2}+n^{2}\right)^{-\beta / 2}(2 \pi i)^{-\beta} c_{m n} e^{2 \pi i(m x+n y)}$ and one has $I_{\alpha} I_{\beta} f=I_{\alpha+\beta} f$. For sufficiently smooth $f, \sum_{m, n}\left(m^{2}+n^{2}\right)^{\beta / 2}(2 \pi i)^{\beta} c_{m n} e^{2 \pi i(m x+n y)}$ is the Fourier series of a function in $L$ which we denote by $I^{\beta} f$ and, automatically, $I_{\beta} I^{\beta} f=I^{\beta} I_{\beta} f=f$. Thus if $\Delta\left(I_{2-\beta} f\right)$ is continuous, where $0 \leqq \beta \leqq 2$ and $\Delta=\partial^{2} / \partial x^{2}+\partial^{2} / \partial y^{2}$, then $f^{\beta}=\Delta\left(I_{2-\beta} f\right)$. As is the case with fractional integration of functions of one variable, there are relations between the function classes to which $f, I_{\beta} f$, and $I^{\beta} f$ belong. For example: (i) If $f=f(x, y) \in L^{p}, p>1, \beta<2 / p$, then $I_{\beta} f \in L^{q}$ where $1 / p-1 / q=\beta / 2$. If $p \geqq 1$ and $2 / p<\beta<2 / p+1$, then $I_{\beta} f \in \operatorname{Lip}(\beta-2 / p)$. (ii) If $f \in \operatorname{Lip} \alpha, 0<\alpha<1$, then $I_{\beta} f \in \operatorname{Lip}(\alpha+\beta)$ for $\alpha+\beta<1$ and $I^{\beta} f \in \operatorname{Lip}(\alpha-\beta)$ for $0<\beta<\alpha$. (Received September 18,1953.)

118. J. K. Hale: Periodic solutions of systems of nonlinear differential equations.

Consider systems of the form (1) $\ddot{x}_{j}+a_{j} \dot{x}_{j}+\sigma_{j}^{2} x_{j}=\epsilon q_{j}(x, \dot{x} ; \epsilon), j=1,2, \cdots, n$, 
where $\sigma_{j}>0, a_{j} \geqq 0$, 6 is a small real parameter, $x=\left(x_{1}, \cdots, x_{n}\right), \dot{x}=\left(\dot{x}_{1}, \cdots, \dot{x}_{n}\right)$, and $q_{j}$ are analytic functions for $|\epsilon|<\epsilon_{0},\left|x_{j}\right|<A,\left|\dot{x}_{j}\right|<A$. A variant of Poincaré's method of casting out the secular terms is studied and the convergence is proven. If $a_{k}=0$ for some $k$, and the equation (2) $\int_{0}^{2 \pi} q_{k}\left(B \sigma_{b}^{-1} \sin t, B \cos t, 0, \cdots, 0 ; 0\right) \cos t d t$ $=0$ has a simple root for some $B=b$, then there is a periodic solution (limit cycle) to (1) of the form $C_{k}: x_{k}=B_{0} \sigma_{k}^{-1} \cos (\tau t+\phi)+\epsilon W_{k}(\tau t+\phi ; \epsilon), x_{j}=\epsilon W_{j}(\tau t+\phi ; \epsilon), j \neq k$, $j=1,2, \cdots, n$, where $\phi$ is an arbitrary constant and all $W_{j}$ are analytic functions of $\epsilon$ with coefficients periodic in $t$, and $\tau=\sigma_{k}+O(\epsilon), B_{0}=b+O(\epsilon)$ are constants. Thus, the limit cycle $C_{k}$ is characterized by a frequency $\tau$ close to $\sigma_{k}$, and by the fact that $x_{k}$ may be large and $x_{1}, \cdots, x_{k-1}, x_{k+1}, \cdots, x_{n}$ are small. In particular, for systems (3) $\ddot{x}_{j}+\sigma_{j}^{2} x_{j}=\epsilon q_{j}(x, \dot{x} ; \epsilon)$, we have $n$ analogous limit cycles $C_{j}$. If we suppose in (1) that $a_{k}=0$ and $q_{k}(x, \dot{x} ; \epsilon)=f\left(x_{k}\right) \dot{x}_{k}+g\left(x_{k}, \dot{x}_{k}\right)+h(x, \dot{x})+\epsilon q^{*}(x, \dot{x} ; \epsilon)$, where $f\left(x_{k}\right)$ $=a_{0}+a_{1} x_{k}^{2}+\cdots+a_{m} x_{k}^{2 m}, a_{0} a_{m}<0, g\left(-x_{k}, \dot{x}_{k}\right)=-g\left(x_{k}, \dot{x}_{k}\right), h\left(x_{k}, \dot{x}_{k}, 0, \cdots, 0\right)=0$, then (2) certainly has a root $B=b$. Furthermore, if one considers (3) with $q_{j}(x,-\dot{x} ; \epsilon)$ $=q_{j}(x, \dot{x} ; \epsilon)$, then there is a periodic solution $C_{b}$ to (3) for every $B,|B|<A$ and for $k=1,2, \cdots, n$ ( $n$ two-dimensional manifolds of cycles). (Received October 13, 1953.)

119t. J. K. Hale: Systems of linear differential equations with L-integrable periodic coefficients.

Consider the system (1) $d^{2} y_{i} / d x^{2}+\sigma_{i}^{2} y_{i}+\lambda \sum_{h=1}^{n} \phi_{i h}(x) y_{h}=0, i=1,2, \cdots, n$, where $\sigma_{1}, \sigma_{2}, \cdots, \sigma_{n}$ are distinct real positive numbers, $\lambda$ is a small real parameter, the $\phi_{i h}(x)$ are real functions, periodic of period $T=2 \pi / \omega, \int_{0}^{T} \phi_{i h}(x) d x=0$, and possessing absolutely convergent Fourier series. By using a variant of Poincare's method of casting out the secular terms in the solutions of the above system, L. Cesari (Mem. Accad. Italia (6) vol. 6, pp. 634-692) proved the following result: If $m \omega \neq \sigma_{i} \pm \sigma_{h}$, $i, h=1,2, \cdots, n, m=0, \pm 1, \pm 2, \cdots$, and if either $\phi_{i h}(x)=\phi_{i h}(-x)$ or $\phi_{i h}(x)$ $=\phi_{h i}(x), i, h=1,2, \cdots, n$, then for $\lambda$ sufficiently small, the solutions of (1) are bounded. In the present study, the same result is obtained by assuming only that the $\phi_{i h}(x)$ are periodic, $L$-integrable, and $\int_{0}^{T} \phi_{i h}(x) d x=0$. A concept of mean value $m[f]$ is discussed for functions of the class $C_{\omega}$ of functions $f=\sum_{i=1}^{k} e^{\alpha_{i}{ }^{x}} \phi_{i}(x)$, with the $\alpha_{i}$ complex constants and the $\phi_{i}(x)$ of period $T, L$-integrable in $[0, T]$. Various theorems are proven concerning $m[f]$ and the primitives $F(x)$ of the functions $f \in C_{\omega}$. In particular, a function $f \in C_{\omega}$ has a primitive $F \in C_{\omega}$ if and only if $m[f]=0$. These theorems are applied in the discussion of the problem above and particularly in the proof of the convergence of the mentioned method of successive approximations under the new conditions. (Received October 13, 1953.)

\section{0t. Israel Halperin: Reflexitivity in function spaces.}

That $L^{p}(B)$ is reflexive if $1<p<\infty$ and $B$ is reflexive has been shown by $R$. S. Phillips, On weakly compact subsets of a Banach space, Amer. J. Math. vol. 65 (1943) pp. 108-136. The $L^{p}(B)$ are a special, nontypical case of the $L^{\lambda}(B)$ spaces discussed in a paper by H. W. Ellis and Israel Halperin, Function spaces determined by a levelling length function, to appear in the Canadian Journal of Mathematics vol. 5 (1953). It is now shown that $L^{\lambda}(B)$ is reflexive if and only if both $L^{\lambda}$ and $B$ are reflexive. (Received September 21, 1953.)

121. W. B. Jurkat: A function theoretic inclusion theorem for Nörlund means. 
Let $N\left(p_{n}\right)$ and $N\left(q_{n}\right)$ be two Nörlund methods with the corresponding power series $p(x)=\sum p_{n} x^{n}, q(x)=\sum q_{n} x^{n}$. For the inclusion $N\left(p_{n}\right) \subseteq N\left(q_{n}\right)$ exact conditions are stated depending only on the singularities of the function $q(x) / p(x)=k(x)$ $=\sum k_{n} x^{n}$ : Suppose that $k(x)$ is regular and $\neq 0$ for $x=0$ and that to each point $\xi \neq 0$ of $|x| \leqq 1$ there exists a real number $\alpha$ such that $k(x)(1-x / \xi)^{\alpha}$ is regular and $\neq 0$ at the point $x=\xi$. Then the following conditions are necessary and sufficient for the inclusion $N\left(p_{n}\right) \subseteq N\left(q_{n}\right)$ with regular $N\left(p_{n}\right)$ : (i) $k(x)$ must be regular for $|x|<1$; (ii) the exponent $\alpha_{0}$ corresponding to $\xi_{0}=1$ must be not negative; (iii) if there are points $\xi_{\mu} \neq 1,\left|\xi_{\mu}\right|=1$, for which the corresponding $\alpha_{\mu}$ are positive, then $\alpha_{0} \geqq \alpha_{\mu}$ must hold for $\mu=1, \cdots, m$. (Points with $\alpha \leqq 0$ are not essential.) By considering the case $p(x)=1$ one sees that (i), (ii), (iii) are at the same time the exact conditions for regularity of $N\left(k_{n}\right)$. (Received October 16,1953 .)

\section{2t. W. B. Jurkat: Questions of signs in power series.}

In extending the well known theorem of Kaluza and Szegö concerning the signs in a reciprocal power series there are obtained some results on the distribution of signs in the quotient $k(x)=\sum k_{n} x^{n}$ of the two power series $q(x)=\sum q_{n} x^{n}$ and $p(x)$ $=\sum p_{n} x^{n}$. Let us set formally $k(x)=q(x) / p(x)$ and $q_{0}=p_{0}=1$. Then it is sufficient for $k_{n} \geqq 0$ resp. $k_{n} \leqq 0$ for all $n \geqq 1$, that the two following conditions are satisfied: $0 \subset p_{n+1} / p_{n} \nearrow, q_{n} / p_{n} /$ resp. $\searrow$. Another but simpler pair of sufficient conditions is $p_{n} \searrow, q_{n}-p_{n} \nearrow$ resp. $\searrow$. There are some more general results using the differences of higher order of $p_{n}$ and $q_{n}$. (Received November 2,1953.)

\section{3t. Konrad Knopp: On the proof of the main Tauberian theorem} for the $C_{k}$ - and $H_{k}$-methods.

For the now classical theorem "If $a_{\nu}$ are real, if $\sum a_{\nu}$ is summable $C_{k}$ or $H_{k}$ to $s$ $(k>0)$, and if $\nu a_{\nu} \leqq M$, then $\sum a_{\nu}=s^{\prime \prime}$ an inductive proof is given which is, for general $k$, of simpler structure than the previous proofs. The main difference from these lies in the fact that the series-to-series transform is used instead of the sequence-to-sequence transform. The proof is especially simple for the $H_{k}$-methods. A remark of Dr. Jurkat is added with respect to a corresponding theorem for general iterated methods. (Received November 3, 1953.)

\section{4t. Jacob Korevaar: $A$ very general form of Littlewood's theorem.}

Let $f(u)$ denote the sum of a power series $\sum a_{n} e^{-n u}$ which is convergent for $u>0$. Littlewood and Hardy have shown that the conditions $a_{n}>-K / n(n=1,2, \cdots)$ and $f(u) \rightarrow s(u \downarrow 0)$ together imply that $s_{n}=\sum_{k \leqq n} a_{k} \rightarrow s(n \rightarrow \infty)$. Now let $L(t)$ denote a slowly oscillating function, that is, a positive continuous function defined for $t>0$ for which $L(c t) / L(t) \rightarrow 1$ as $t \rightarrow \infty$ for every $c>0$. Let $\alpha$ be real, and denote $t^{\alpha} L(t)$ by $\phi(t)$. Let $\omega(u)$ be defined, positive and bounded for $u>0$, while $\omega(u) \downarrow 0$ as $u \downarrow 0$. The following general Tauberian theorem is proved. The conditions $a_{n}>-\phi(n)$ $(n=1,2, \cdots)$ and $|f(u)-s|<\omega(u) \quad(u>0)$ together imply that $\left|s_{n}-s\right| \leqq \rho(n)$ $(n=0,1, \cdots)$, where $\rho(0)=K_{1}, \rho(n)=\min _{p-1,2, \ldots}\left\{K_{2} n \phi(n) / p+K_{3}^{p} \omega(p / n)\right\}(n \geqq 1)$ if $\lim \inf _{u+0} u \log \omega(u)>-\infty$, and $\rho(n)=0(n=0,1, \ldots)$ if $\lim \inf _{u+0} u \log \omega(u)$ $=-\infty$. Here the numbers $K_{i}$ depend only on the functions $\phi$ and $\omega\left(K_{3}>1\right)$. It is shown by various examples that the above estimates are essentially best possible. For example, if $\omega(u)=\exp (-1 / u)$, then $a_{n}>-o\left(n^{-1 / 2}\right)$ implies convergence of $\sum a_{n}$. However, the function $f(\log 1 / x)=\int_{x}^{1}(1-x)^{-2}\left\{1-2 x+2 x^{4}-2 x^{9}+\cdots\right\} d x$ is $O\{\exp (-1 /(1-x))\}$ as $x \uparrow 1$ while the corresponding series $\sum a_{n}$ for which $a_{n}$ 
$=O\left(n^{-1 / 2}\right)$ is easily seen to diverge. The above results improve and complete earlier results of the author [Duke Math. J. vol. 18 (1951) pp. 723-734; Proc. Nederl. Akad. Wetensch. Ser. A vol. 56 (1953) pp. 281-293]. (Received September 28, 1953.)

125. Jacob Korevaar: Another numerical Tauberian theorem for power series.

The power series associated with the series $\sum a_{n}:(0)+1-1 / 2+1 / 3-1 / 4+\cdots$ is $f(u)=\sum(-1)^{n-1} n^{-1} e^{-n u}=\log \left(1+e^{-u}\right)$. Clearly $|f(u)-\log 2|<u(u>0)$, and $a_{n} \geqq-1 / n$. Thus the Tauberian theorem of the preceding paper gives the estimate $\left|s_{n}-\log 2\right|$ $\leqq C / \log n(n \geqq 2)$. A much stronger result, however, is known in this case; one actually has $\left|s_{n}-\log 2\right| \leqq C / n(n \geqq 1)$. A similar stronger result holds whenever $f(u)=\sum a_{n} e^{-n u}$ (where the series is assumed to converge for $u>0$ ) is analytic at $u=0$. This hypothesis together with the condition $a_{n} \geqq-\phi(n)=-n^{\alpha} L(n), L(n)$ a slowly oscillating function, implies that $\left|s_{n}-f(0)\right| \leqq C \phi(n)(n \geqq 1)$. A more general result is the following. Let the series $f(u)=\sum a_{n} e^{-n u}$ converge for $u>0$, and let there be a function $g(u)$ analytic at $u=0$ such that $|f(u)-g(u)| \leqq \omega(u)$ on some interval $0<u<\delta$, where $\omega(u) \downarrow 0$ as $u \downarrow 0$. Let $a_{n} \geqq-\phi(n)$. Then $\left|s_{n}-g(0)\right| \leqq \rho(n)=\min _{p}\left\{C_{1} n \phi(n) / p\right.$ $\left.+C_{2}^{p} \omega(p / n)\right\} \quad\left(n \geqq n_{0}\right)$, where the minimum is taken over all $p$ satisfying $C_{3} \leqq p \leqq C_{4} n$. In case $\lim \inf _{u v} u u \log \omega(u)=-\infty$ one has the stronger conclusion $f(u) \equiv g(u)$, hence $\left|s_{n}-g(0)\right| \leqq C_{5} \phi(n)$. The proofs of these results depend upon a further refinement of the theory of best $L_{1}$ approximation from one side by polynomials of given degree as developed by Geza Freud and by the author. (Received November 13, 1953.)

126. M. Z. Krzywoblocki: On some peculiarities of partial differential systems in two independent variables.

By means of a non-one-to-one transformation a system of partial differential equations in two independent variables is transformed onto an ordinary differential system with a parameter. The following lemma was proved: Each of all the curves lying in the intersections of each of all the integral surfaces of a system of nonlinear partial differential equations of the $k$ th order in two independent variables with the planes perpendicular to $x$ - (or $y$-) axis represents a singular invariant solution of the corresponding differential form of this system, which solution is invariant under the $k$-enlargement of some one-parameter continuous group of transformations with one pair of independent variables being variables and the other being parameters. These curves represent the peculiar solutions of the system in question and they are some extremities on the integral surfaces. (Received October 9, 1953.)

127. R. E. Lane: The integral of a function with respect to a function. II.

Infinite subdivisions (and refinements of infinite subdivisions) are used with the trapezoidal formula to generalize the author's previous definition of $\int_{a}^{b} u(x) d v(x)$, still retaining integration by parts and other properties of the Stieltjes integral. An example is given in which each of $u$ and $v$ is totally discontinuous. If $u$ is bounded and Lebesgue integrable and $v(x)=x$ in $[a, b]$, then $\int_{a}^{b} u(x) d v(x)$ is equal to the Lebesgue integral. (Received October 13, 1953.)

128. Norman Levinson and J. J. Levin (p): Singular perturbations of nonlinear systems of differential equations and an associated boundary layer equation. 
The relationship of the solutions of a certain "full" system of differential equations (1) as $\epsilon \rightarrow 0+$, to a particular solution of a related "degenerate" system (2) is investigated. The systems are: (1) $d x / d t=f(x, y, t, \epsilon), \epsilon^{r} d y / d t=g(x, y, t, \epsilon) ;(2) d x / d t$ $=f(x, y, t, 0), 0=g(x, y, t, 0)$; where $x$ and $f$ are real vectors of dimension $n_{0} \geqq 1, y$ and $g$ are real vectors of dimension $n \geqq 1, \epsilon>0$, and $r>0$ and fixed. Systems containing higher powers of $\epsilon$ and more dependent variables are also investigated. A "boundary layer equation" related to (1) is discussed. The results generalize those of Tihonov (Mat. Sbornik N.S. vol. 27 (1950) pp. 147-156). (Received October 13, 1953.)

\section{G. G. Lorentz: The Borel property of summability methods.}

For a function $\Omega(n)$ tending to $+\infty$ with $n$, and a regular summability method $A$, the following statements are equivalent: (1) Each sequence $s_{n}$ with $s_{1}+\cdots+s_{n}$ $=o(\Omega(n))$ is $A$-summable; (2) for each convergent series $\sum c_{n}, A-\lim c_{n} \Omega(n)=0$; (3) $R\left(\lambda_{n}, 1\right) \subset A$, where $\lambda_{n}=\exp \left(\Omega(1)^{-1}+\cdots+\Omega(n)^{-1}\right)$. For this, $\sum_{v} \Omega(v)\left|\Delta_{v} a_{n v}\right|$ $=O(1)$ is necessary and sufficient. It is written then $\Omega(n) \in S_{2}(A)$. Such $\Omega$ were discussed by the author [Canadian Journal of Mathematics vol. 1 (1949) pp. 305-319; vol. 3 (1951) pp. 236-256]. They are applied here in the following way: $A$ has the Borel property if there is an $\Omega \in S_{2}$ with $\sum \exp (-\delta \Omega(n))<+\infty$ for each $\delta>0$. For some special methods, necessary and sufficient conditions are given. Also, $A$ has the "Banach property" if $A$-lim $\Phi_{n}(x)=0$ a.e. for each orthonormal system $\Phi_{n}(x)$. Sufficient for this is that there exists an $\Omega \in S_{2}(A)$ with $\sum \Omega(n)^{-1} \log ^{2} n<+\infty$. (Received October 14, 1953.)

130t. K. S. Miller and L. A. Zadeh: On an integral equation occurring in the theory of prediction.

In the theory of prediction one frequently encounters the integral equation $f(t)$ $=\int_{0}^{T} R(t, \zeta) g(\zeta) d \zeta$ where $t$ ranges in the interval $[0, T]$ and the kernel $R$ is of the form $R(t, x)=\int_{-\infty}^{\infty} M_{\zeta} \bar{H}(t, \zeta) M_{\zeta} \bar{H}(x, \zeta) d \zeta$. Here $M_{\zeta}$ is a linear differential operator operating on the variable $\zeta$ and $\bar{H}$ is essentially the one-sided Green's function of some other linear differential operator. A solution of the integral equation is obtained and the results discussed and interpreted in terms of prediction theory. (Received September 29, 1953.)

\section{1t. V. L. Shapiro: Cantor-type uniqueness of multiple trigono-} metric integrals.

Let $c(u)$ be a complex-valued function integrable on every bounded domain in $n$-dimensional euclidean space $E_{n}$, and for which $c(u)|u|^{-2}$ is in $L_{1}$ on $E_{n}$. Suppose that the integral $\int_{E_{n}} e^{i x}{ }^{c} c(u) d u$ is spherically summable $(C, 1)$ to zero almost everywhere and that the $(C, 1)$ spherical mean of this integral of rank $R, \sigma_{R}^{(1)}(x)$, is such that $\lim \sup _{R \rightarrow \infty}\left|\sigma_{R}^{(1)}(x)\right|<\infty$ in $E_{n}-Z$ where $Z$ is a closed set of vanishing capacity. It is then shown in this paper that $c(u)$ is equal to zero almost everywhere. The proof is brought about by considering generalized Laplacians of the first kind in connection with Riemann summability of trigonometric integrals. Further results of the above kind are obtained when $c(u)|u|^{-2}$ is in $L_{2}$ on $E_{n}$ and almost everywhere $(C, 1)$ summability is replaced by summability in $E_{n}-Z$. In particular for the plane, it is shown that if $c(u)=O\left(u^{1-\epsilon}\right), \epsilon>0$, is integrable on every bounded domain, if $\int_{E_{n}} e^{i x u} c(u) d u$ is spherically summable $(C, 1)$ to zero in $E_{2}-Z$, and if $\int_{E_{2}-D_{2}(0,1)} e^{i x u} c(u) u^{-2} d u$ is spherically convergent to a continuous function in $E_{2}-W$ where $W$ is a closed de- 
numerable set such that $Z W=0$, then $c(u)$ is equal to zero almost everywhere. (Received October 13, 1953.)

\section{2t. H. S. Wall: Concerning harmonic matrices.}

The statement that the $n \times n$ matrix $M$ is a harmonic matrix means that $M_{i j}$ is a complex-valued function from the sensed pairs $(x, y)$ of real numbers which, for each number $y(x)$, is continuous and of bounded variation in $x(y)$ on every interval, and, for each $x, y, z, M(x, y) M(y, z)=M(x, z)$ and $M(x, x)=I$. Theorem $\mathrm{A}$. The $n \times n$ matrix $M$ is harmonic if and only if there exists an $n \times n$ matrix $F$ of complexvalued functions from the real numbers, continuous and of bounded variation in every interval, such that $F(0)=0$ and $M(x, y)=I+\int_{x}^{y} d F(s) \cdot M(s, y)$. This correspondence $M \leftrightarrow F$ is reversible, $M_{i j}(x, y)$ is continuous in $(x, y)$ and $M(x, y)=I+\int_{x}^{y} M(x, s)$ - $d F(s)$. Theorem B. If $n=2$, det $M(x, y)=1$ if and only if $F_{11}=-F_{22}$. Theorem C. Suppose $f$ is an $n$-rowed column vector of functions and $z$ an $n$-rowed column vector of numbers. The equation $f(x)=z+\int_{x}^{y} d F(s) \cdot f(s)$ has the unique solution $f(x)=M(x, y)$ - $z$. If $n=2$, the quotient $f_{1} / f_{2}$ is the unique solution $w$ of the Stieltjes integral equation $w(x)-w(y)=\int_{y}^{x} w^{2} d F_{21}+\int_{y}^{x} w d\left(F_{22}-F_{11}\right)-\int_{y}^{x} d F_{12}$. (Received August 28, 1953.)

\section{Daniel Waterman: On an integral of Littlewood and Paley.}

Littlewood and Paley defined $g(\theta)=\left\{\int_{0}^{1}(1-\rho)\left|\phi^{\prime}\left(\rho e^{i \theta}\right)\right|^{2} d \rho\right\}^{1 / 2}$ for $\phi(z)$ of class $H_{p}$, $p \geqq 0$. They showed that $\|g(\theta)\|_{p} \leqq A_{p}\left\|_{\phi}\right\|_{p}$. For $p \geqq 1$ they showed the converse result, $\|\phi\|_{p} \leqq A_{p}\|g\|_{p}$. Here both results are shown to hold for the Hille-Tamarkin class $\mathfrak{W}_{p}$ in the half-plane, with the definition $g(\tau)=\left\{\int_{0}^{\infty} \sigma\left|\phi^{\prime}(\sigma+i \tau)\right| d \sigma\right\}^{1 / 2}$, superseding the result $\|g(\tau)\|_{p} \leqq A_{p}\left\|_{\phi}\right\|_{p}$ previously given by this author for the definition $g(\tau)=\left\{\int_{0}^{\infty}\left(1-e^{-\sigma}\right)\left|\phi^{\prime}(\sigma+i \tau)\right|^{2} d \sigma\right\}^{1 / 2}$. (Received October 13, 1953.)

\section{F. M. Wright: A transformation for S-fractions.}

It is proved that, if the formal power series $Q(w)=\sum_{n=0}^{\infty} \mu_{n} w^{n+1}$ and $P(w)$ $=\sum_{n=0}^{\infty} \lambda_{n} w^{n+1}$ are both the corresponding power series of $S$-fractions, then $P(w)$ $\equiv \lambda_{0} w+w Q(w)$ if and only if there is a set of parameters in terms of which the $S$ fraction expansions of $Q(w)$ and $P(w)$ have certain prescribed forms. This result is then used to obtain results relative to the backward extension of Stieltjes and Hausdorff moment sequences. Many of the results developed relative to the backward extension of a given Stieltjes moment sequence $\left\{\mu_{n}\right\}$ such that the $S$-fraction expansion of the formal power series $\sum_{n-0}^{\infty} \mu_{n} w^{n+1}$ is nonterminating have been proved previously by H. S. Wall [Trans. Amer. Math. Soc. vol. 31 (1929) pp. 91-116], but it is believed that the present development, which avoids the use of determinants, is simpler and more direct. (Received October 1, 1953.)

\section{5t. R. E. Zink: Direct unions of measure spaces.}

Let $\left\{\left(Y_{x}, \Sigma_{x}, \nu_{x}\right)\right\}$ be a family of measure spaces such that the $Y_{x}$ are pairwise disjoint point sets and such that the parameter $x$ ranges over the set $X$ of the measure space $(X, \Sigma, \mu)$. Then if $(X, \Sigma, \mu)$ is a $\sigma$-finite measure space, and if the function $\nu_{x}\left(Y_{x}\right)$ is defined for all $x$, finite valued, and measurable $(\Sigma)$, it is possible to construct a $\sigma$-ring of subsets of the space $S \equiv \cup_{x \in X} Y_{x}$ and a measure, $\tau$, on this $\sigma$-ring. Furthermore the resulting measure space, which is called the direct union of $\left(Y_{x}, \Sigma_{x}, \nu_{x}\right)$ with respect to $(X, \Sigma, \mu)$, has the property that if $E$ is an arbitrary measurable set, $E_{x}$ belongs to $\Sigma_{x}$ for almost all $x$, the function $\nu_{x}\left(E \cap Y_{x}\right)$ is, when arbitrarily defined on the remaining null set, measurable $(\Sigma)$ and $\tau(E)=\int \nu_{x}\left(E \cap Y_{x}\right) d \mu(x)$. The method of 
construction is also applicable to the cartesian product of two measure spaces and it is possible by this technique to establish an extension of the Lebesgue planar measure. In connection with the above, a theorem on integration in spaces which are related to direct unions of measure spaces can be established. (Received October 13, 1953.)

\section{Applied Mathematics}

\section{R. L. Evans: REAC solution in the large of a linear differential} equation.

Under Office of Ordnance Research Contract DA-11-022-ORD-489 the differential equation (1) $0=y^{(2)}-y\left(3 / 8 \alpha^{2}+1 / \alpha^{2} r^{11}-11 / 8 \alpha^{2} r^{8}-(2 \lambda+1) / 2 \alpha+k(k+1) / r^{2}\right)$ was studied. Eigenvalues were sought which would lead to solutions of $(1) \ni y(0)=y(+\infty)$ $=0$. Since 0 and $\infty$ are both irregular singular points of (1) this involves solution in the large-the interrelation of solutions about different singular points. The case in which $k=1$ and $\alpha=.01$ was treated on the Reeves Electronic Analog Computer (REAC) as follows. Let $y_{1}(1)=1, y_{1}^{\prime}(1)=0, y_{2}(1)=0, y_{2}^{\prime}(1)=1, \lim _{r \rightarrow+\infty}\left(y_{3} \& y_{3}^{\prime}\right)=0$, and $\lim _{r \rightarrow+\infty}\left(y_{4} \& y_{4}^{\prime}\right) \neq 0$; and let (2) $R_{1}(\lambda) \cdot y_{1}+y_{2}=$ (multiple of $\left.y_{3}\right)$ define $R_{1}(\lambda)$. Let $r=1 / x, y(r)=Y(x), \lim _{x \rightarrow+\infty}\left(Y_{1} \& Y_{1}^{\prime}\right)=0, \lim _{x \rightarrow+\infty}\left(Y_{2} \& Y_{2}^{\prime}\right) \neq 0$, and let (3) $R_{2}(\lambda) \cdot y_{1}+y_{2}=$ (multiple of $Y_{1}$ ) define $R_{2}(\lambda)$. Desired $\lambda$ 's are $\ni R_{1}(\lambda)=R_{2}(\lambda)$. In (2) (or (3)) a term in $y_{4}$ (or $Y_{2}$ ) is also present except when $R_{1}$ (or $R_{2}$ ) is $\ni$ the extra term changes sign. These values are determined by trial and error on the REAC but are continuous functions of $\lambda$. Values for this case are: $(3 / 8-(2 \lambda+1) / 200):(.2, .05, .01)$; $R_{1}:(-2.26,-4.58,-11.36)$; and $R_{2}:(-.077,-.92,-2.15)$ in corresponding sequence. The REAC method is the best exploratory one but may need supplementing at near-critical eigenvalues (small values in the first sequence above). Also, for the case treated here no satisfactory eigenvalue was found. The method can be generalized. (Received October 15, 1953.)

\section{7t. H. E. Salzer: Osculatory quadrature formulas.}

By integrating $f(x)$ in Hermite's $n$-point osculatory interpolation formula (1) $f\left(x_{0}+p h\right)=\sum_{i=-[(n-1) / 2]}^{[n / 2]}\left\{L_{i}^{(n)}(p)\right\}^{2}\left\{1-2 L_{i}^{(n)}(i)(p-i)\right\} f_{i}+\sum_{i=-[(n-1) / 2]}^{[n / 2]}\left\{L_{i}^{(n)}(p)\right\}^{2}$ $\cdot(p-i) h f_{i}^{\prime}+R_{2 n}(p)$, where $x=x_{0}+p h, x_{i}=x_{0}+i h, f\left(x_{i}\right) \equiv f_{i}, f^{\prime}\left(x_{i}\right) \equiv f_{i}^{\prime}$ and where (2) $L_{i}^{(n)}(p)=\prod_{j=-[(n-1) / 2]}^{\prime(n / 2)}\{(p-j) /(i-j)\}, \quad j=i$ absent from $\Pi^{\prime}$, and (3) $R_{2 n}(p)$ $=f^{(2 n)}(\xi) h^{2 n}\left\{\prod_{j--[(n-1) / 2]}^{[n / 2]}(p-j)\right\}^{2} /(2 n) !, x_{-[(n-1) / 2]} \leqq \xi \leqq x_{[n / 2]}$, one obtains an extremely accurate series of integration formulas which utilize both the integrand and its first derivative at $n$ fixed equally spaced points. They are of the form (4) $\int_{x_{0}+r h}^{x_{0}+e h} f(x) d x$ $=\sum_{i=-[(n-1) / 2]}^{[n / 2]}\left\{h A_{i} f_{i}+h^{2} B_{i} f_{i}^{\prime}\right\}+R$. These $n$-point "osculatory quadrature formulas" are not only exact when $f(x)$ is a polynomial of degree $\leqq 2 n-1$, but can also be shown to be much more accurate than a $2 n$-point formula which is obtained by integrating Lagrange's formula and which employs the integrand alone at equally spaced arguments at intervals of $h$. The exact fractional values of $A_{i}$ and $B_{i}$ are tabulated for $n=2(1) 7$ and for every combination of $(r, s)$ within the range $(-[(n-1) / 2],[n / 2])$. A supplementary schedule tabulates the exact values of the coefficients $C(n, r, s)$ in the remainder $R=C(n, r, s) h^{2 n+1} f^{(2 n)}\left(\xi_{1}\right), x_{-[(n-1) / 2]} \leqq \xi_{1} \leqq x_{[n / 2]}$, which holds whenever $f^{(2 n)}(\xi)$ in (3) is a continuous function of $p$. The coefficients $A_{i}$ and $B_{i}$ are shown to satisfy $A_{-[(n-1) / 2]+j}, B_{-[(n-1 / 2)+j}$ for interval $(-[(n-1) / 2]+k,-[(n-1) / 2]+k+m)$ $=A_{[n / 2]-i},-B_{[n / 2]-i}$, resp. for interval $([n / 2]-k-m,[n / 2]-k)$ for every $n$, $1 \leqq m \leqq n-1, j=0,1, \cdots, n-1$. (Received October 2, 1953.) 


\section{GEOMETRY}

\section{R. B. Deal: Union differentiation and union correspondence.}

Recently, T. K. Pan has stated in an abstract certain results for what he terms relative geodesics. It is shown in the present work that his relative geodesics are union curves and that what he refers to as relative first curvature is what Springer and other authors have called union curvature. Some additional generalizations of well known results for geodesics are obtained for union curves. For instance, the limit of the quotient of the angle between the union curves tangent to a given curve at two points and the arc length between these points is shown to be the union curvature of the curve. Generalized intrinsic derivatives are defined in two different manners. Properties of each are studied. A generalization of the theorem stating that the divergence of the unit normal to a curve in a surface is the negative of the geodesic curvature is obtained for union curvature. Two theorems are obtained for union correspondence. The first gives a set of necessary and sufficient conditions for two surfaces to be in union correspondence, whereas the second theorem extends the analysis of the first in order to find conditions on the congruence which allow the existence of a second surface whose geodesics are in union correspondence with union curves on the first surface. (Received October 14, 1953.)

\section{P. C. Hammer: Maximal convex sets.}

Let $L$ be an arbitrary linear vector space over the real or complex numbers. A set $S$ is a semispace at the origin $\theta$ of $L$ if and only if (a) $S$ does not contain $\theta$, (b) $S$ is convex, (c) $S$ contains one of the open half-lines determined by $\theta$ on every line through $\theta$. All semispaces are obtained by translations. The semispaces with $L$ form the minimal intersection basis for all convex sets in $L$. If $C_{0}$ and $C_{1}$ are nonvacuous disjoint convex sets in $L$, then there exists a dichotomy of $L$ into convex sets $H_{0}, H_{1}$, such that $H_{0} \supset C_{0}$ and $H_{1} \supset C_{1}$. Every such $H_{0}$ is an intersection set of a class of translates of a semispace. In degenerate cases the boundary of a semispace is a hyperplane. (Received September 24, 1953.)

\section{LOGIC AND Foundations}

\section{J. C. E. Dekker: $A$ theorem on hypersimple sets.}

This paper gives an affirmative answer to the following question raised by Post: "Can a creative set be Turing reducible to a hypersimple set?" Let $E$ be the class of all recursive sets and $F$ the class of all recursively enumerable sets. " $\alpha$ t-red $\beta$ " denotes: $\alpha$ is reducible to $\beta$ by truth tables; " $\alpha$ red $\beta$ " denotes: $\alpha$ is Turing reducible to $\beta$. Two sets are called Turing equivalent if they are Turing reducible to each other. Theorem. For every set $\alpha \in F-E$ one can effectively find a set $\beta$ such that: (a) $\beta$ is hypersimple, (b) $\alpha$ red $\beta$ and $\beta$ red $\alpha$. Corollary 1. There exist sets $\sigma$ and $\tau$ such that $\sigma$ red $\tau$ is true, but $\sigma$ t-red $\tau$ is false. Corollary 2. There exist two sets in $F-E$ which are not Turing equivalent if and only if there exist two hypersimple sets which are not Turing equivalent. The proof of part (a) does not use the fact that hypersimple sets exist. As a side result a new proof for the existence of a hypersimple set is therefore obtained. (Received October 13, 1953.)

141t. David Nelson: Contraposition with strong negation. Preliminary report. 
A predicate calculus and arithmetic are presented which make a further separation of constructible concepts beyond that of P-realizable systems (J. Symbolic Logic vol. 14 (1949) pp. 16-26). A truth definition is given. A formula $A \supset B$ is $P$-realizable just in case there exist a pair of partial recursive functions $\phi$ and $\psi$ such that, if $a P$-realizes $A$, then $\phi(a) \mathcal{P}$-realizes $B$, and if $b \mathcal{P}$-realizes $B$, then $\psi(b) \mathcal{P}$-realizes $A$. Other clauses are as for P-realizability. A predicate calculus shown to satisfy the definition is obtained from Schütte's $K_{3}$ (Math. Ann. vol. 122 (1950) pp. 47-65) by replacing "Zusammenziehungen" by the weak rule " $A \supset(A \supset B)$ is deducible from $A \supset(A \supset(A$ $\supset B)$ )," adding an inference rule and axioms (including contraposition) for strong negation, and a modification of the right hand $\mathrm{V}$ and $\exists$ introduction rules. The arithmetic system replaces the induction schema by a rule of inference. The nonprovability of certain formulas is established. The new systems enjoy symmetries not found in the previously discussed constructive ones. A further paper in preparation establishes a kind of extensionality and discusses deductive extent of the systems. (Received October 13, 1953.)

\section{Statistics and Probability}

142. H. D. Brunk: The strong law of large numbers for asymptotically almost periodic stochastic processes. Preliminary report.

The concept of a stochastic process whose random variables have an asymptotically almost periodic joint distribution is introduced, generalizing the concept of a stationary stochastic process. Doob (Duke Math. J. vol. 6 (1940) pp. 290-307) adapted Birkhoff's individual ergodic theorem to obtain a strong law of large numbers for stationary processes. A strong law of large numbers is obtained for asymptotically almost periodic stochastic processes using the individual ergodic theorem as generalized by Riesz (Comment. Math. Helv. vol. 17 (1944-45) pp. 221-239) and an invariant measure (Dowker, Duke Math. J. vol. 14 (1947) pp. 1051-1061) based on averages (Dowker, loc. cit., Dunford and Miller, Trans. Amer. Math. Soc. vol. 60 (1946) pp. 538-549). Examples are given of asymptotically almost periodic processes. (Received October 9, 1953.)

\section{TOPOLOGY}

\section{3t. L. E. Pursell: On multiplicative semigroups of non-negative semicontinuous functions.}

The set of all non-negative upper semicontinuous functions on a topological space $X$ with the multiplication operation forms a semigroup $U(X)$. The idempotents of $U(X)$ are those functions on $X$ which vanish on an open set and are one on its complement. The nonzero idempotents of $U(X)$ are ordered as follows: $f>g$ if $f \cdot g=g$. $M(U, X)$ consists of all minimal nonzero idempotents with the topology: $A \subset M(U, X)$ is closed if $A$ consists of all $g$ in $M(U, X)$ which precede some idempotent $f$. If $X$ is a $T_{1}$ topological space, then $X$ is homeomorphic to $M(U, X)$. If $X$ and $Y$ are $T_{1}$ spaces and $U(X)$ and $U(Y)$ are isomorphic, then $X$ and $Y$ are homeomorphic. One can obtain similar results for the multiplicative semigroups of all non-negative lower semicontinuous functions, all bounded non-negative lower semicontinuous functions, and all bounded non-negative upper semicontinuous functions. (Received October 16, 1953.)

144t. C. T. Yang: Topological generalizations of theorems of BorsukUlam, Kakutani-Yamabe-Yujobob and Dyson. 
Let $X$ be a compact Hausdorff space and let $T$ be an involution on $X$ without fixed point such that there is some element of the $n$th special homology group of $(X ; T)$ in the sense of Smith (Lefschetz, Algebraic topology, Appendix B), which is not annihilated by any homomorphism induced by a map of $(X ; T)$ into an arbitrary $T$ space $(Y ; T)$. Let $E, F$ be closed subsets of $X^{2}$ such that (i) $E \cup F=X^{2}$, (ii) $(x, y) \in E$ $\leftrightarrow(y, x) \in E \leftrightarrow(T(x), y) \in F \leftrightarrow(x, T(y)) \in F$, (iii) $\{(x, x): x \in X\} \subset E-F$. Two points $x, y$ of $X$ are called orthogonal if $(x, y) \in E \cap F$. It is proved that a continuous realvalued function on $X$ maps some $n$ mutually orthogonal points of $X$ together with their $T$-images into a single value. This result is topological and generalizes a previous generalized Dyson's theorem of the author (Bull. Amer. Math. Soc. Abstract 59-6720). The proof is roughly as follows: The nerves of certain finite open coverings of $X$ are imbedded into euclidean spaces in such a way that the previous generalized Dyson's theorem can apply. Then a limit procedure yields the desired assertation. Topological generalizations of this kind of Borsuk-Ulam's and Kakutani-Yamabe Yujob6's theorems are also established. (Received October 7, 1953.)

\author{
F. J. WeYL, \\ National Research Council \\ J. W. T. Youngs, \\ Associate Secretary
}

